\title{
A reinterpretation of extinction in discriminated avoidance'
}

\author{
D. GENE DAVENPORT and RICHARD D. OLSON, Saint \\ Louis University, St. Louis, Missouri 63103
}

Accepting escape from the warning signal and/or avoidance of shock as the source of reinforcement for the discriminated avoidance response, extinction is redefined in terms of withholding these effects following the response. The effects of warning intervals of 5,10 , and $20 \mathrm{sec}$ on the redefined extinction procedure were reported, and rapid reliable decreases in responding were found for all Ss, similar to positive reinforcement studies.

Most adherents of a two-process theory of discriminated avoidance learning would agree that there is a classical conditioning component in which the neutral warning signal becomes aversive and an instrumental conditioning component involving a response-reinforcement contingency. Thus, although the presentation of shock is the reinforcing event for classical conditioning of fear, escape from the warning signal and/or omission of shock are assumed to be the reinforcing events in the acquisition of the instrumental response (Rescorla \& Solomon, 1967).

In spite of the above, extinction of the avoidance response has in the past been studied by removing the shock and observing the decreasing tendency for the response to occur. Although this may extinguish fear, it is here argued that removing shock following avoidance training is no more extinction of the instrumental avoidance response than the decreased tendency for Ss to respond when satiated for food is extinction in the positive reinforcement situations.

If extinction involves the withholding of reinforcement, a basic change in procedure seems essential. In discriminated avoidance with escape, extinction of the instrumental avoidance response would be expected to occur not when the shock was removed (fear extinction), but when the shock was no longer avoidable, and the warning signal not escapable until the shock occurred. In this case, the drive state, fear, is left intact; but the instrumental response, being ineffective, should extinguish in the absence of reinforcement. Extinction as used here should also be distinguished from what might be called suppression procedures, such as used by Katzev (1967) where, with shock withheld, responding extended the warning signal. Thus, the present argument suggests the need to distinguish between the use of the instrumental response as an index of the underlying motivational state, as in the extinction of acquired drives, and the study under stable drive conditions of the acquisition and extinction of the instrumental response per se. The problem of the present research was to study the effects of various avoidance intervals on the acquisition and redefined "extinction" of the instrumental avoidance response.

Subjects

The Ss were 30 experimentally naive, female Sprague-Dawley albino rats obtained from the colony at the St. Louis University Medical School. All Ss were 98 days old at the start of the experiment and weighed between $180-260 \mathrm{~g}$.

Apparatus

A standard single-lever Gerbrands operant conditioning box was used as the testing chamber with the appropriate programming and recording equipment housed in a separate room. Electric shock used as the aversive stimulus was applied to the grid floor from a Lehigh Valley constant current shocker.

Procedure

Three experimental groups, of five Ss each, were given discriminated avoidanceescape training with either a 5,10 , or $20 \mathrm{sec}$ avoidance interval.
A lever response during the light-buzzer warning signal terminated the signal and prevented the $.4 \mathrm{~mA}$ shock from occurring; or, if not avoided, the same response terminated the shock. A "classical conditioning" escapeonly control group was provided for each of the above warning signal durations, five Ss per group, as an indication of the nonrewarded "operant" level performance to be expected when there is widely differing opportunity to respond and as a baseline to which experimental Ss would be expected to extinguish under the given warning duration when the avoidance contingency was removed. This is a control similar to that independently recommended by D'Amato (1967). Experimental Ss received two days of acquisition and one day of extinction (revised definition), 200 trials per day with an average intertrial interval of $22.5 \mathrm{sec}$. The controls were under the escape-only "extinction" condition all three days. The study was run in five complete replications of six Ss each, one S per condition in each replication.

RESULTS AND DISCUSSION

The primary data reported are per cent anticipatory response over blocks of 40 trials, analyzed by a mixed analysis of variance for two reinforcement conditions (avoidance-escape vs escapeonly) and three warning durations $(5,10$, and $20 \mathrm{sec})$ as between-group variables, and stages of training ( 10 blocks of acquisition and five blocks of ex tinction trials) as a within-group variable. Figure 1 illustrates the acquisition and extinction performances for the six groups. The superior performance of the avoidance Ss over the escape-only controls is significant $(F=43.93, p<.01)$ as is the interaction involving the increase in these differences over Stages of Training $(F=20.49, p<.01)$. Although the main effect of avoidance duration was not significant, the interaction of Duration by Stages is $(F=4.35$, $p<.01)$, indicating the reliability of the apparent early advantage of the $20 \mathrm{sec}$ duration. The main effect of Stages of Training

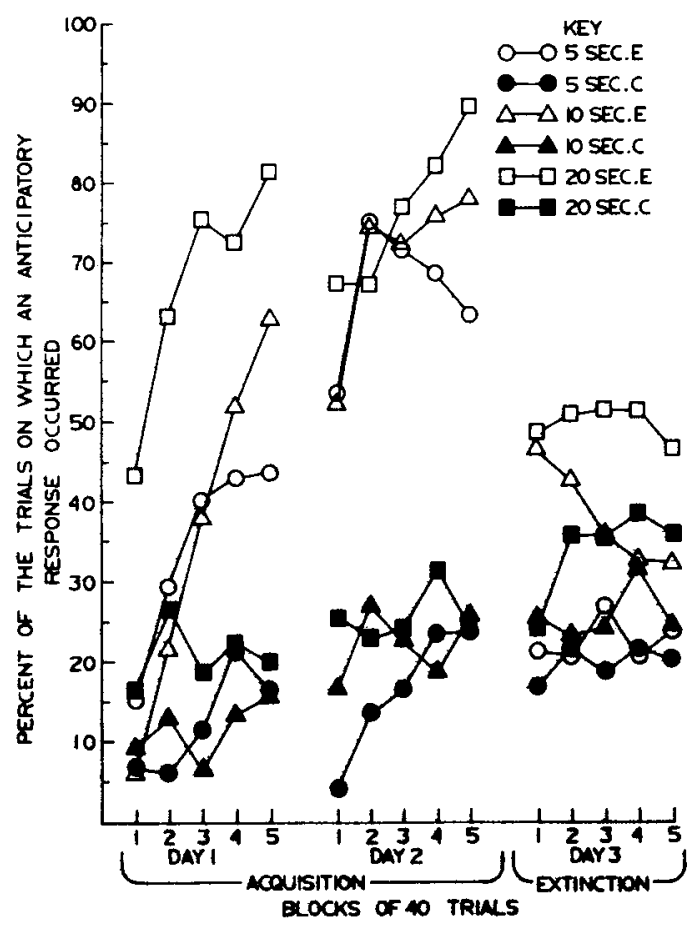

Fig. 1. Mean per cent of the trials on which an anticipatory response occurred in blocks of $\mathbf{4 0}$ trials for avoidance-escape experimental Ss and escape-only controls during acquisition and extinction, presented separately for the 5,10 , and 20 sec warning intervals. 


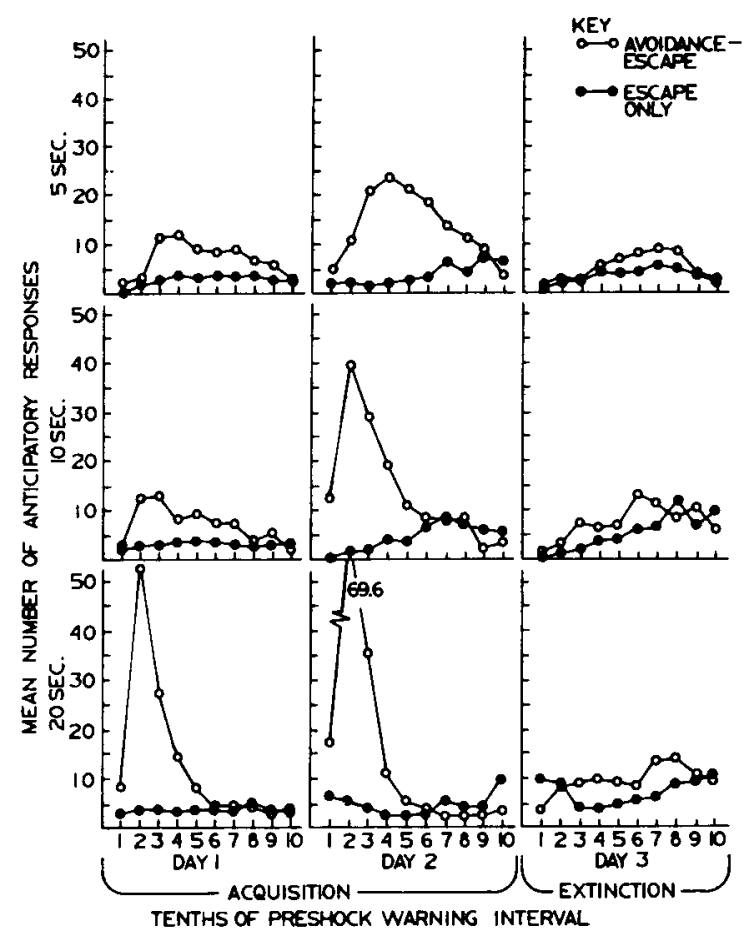

Fig. 2. Mean latency distributions for avoidance-escape $S s$ and escape-only $S s$ in tenths of the respective 5,10 , or 20 sec warning interval for two days of acquisition and one day of extinction.

was highly significant $(F=50.33, p<.01)$. There was no interaction between Reinforcement Conditions and Warning Duration.

The analysis of the extinction data indicated that there was a residual superiority of the avoidance-trained Ss still present during extinction $(F=38.46, p<.01)$. This training effect plus the nonsignificance of any effects on the last block of 40 extinction trials indicated that extinction was complete within 200 trials, with most of the change occurring in the early stages of extinction.

Except for the poor performance of a few Ss in the first replication, the means for the various groups are quite representative of the performances of individual Ss making up a particular group. This relatively poor performance was likely due to starting this group too soon after arrival in the laboratory. In both the acquisition and extinction data, there was a significant Replication by Stages interaction $(F=3.98, p<.05)$, but the main effect of Replications was not significant.

The present results are quite similar to the classical study by Brogden, Lipman, \& Culler (1938) comparing classical and instrumental avoidance conditioning. Sheffield (1948) interpreted the inferior performance of the classical conditioning group not as critical of contiguity theory, but due to the individual differences in the "unconditioned" response to shock, some running and some freezing in the nonavoidance group. The running or freezing response was assumed to be passed forward to the CS through contiguity and when the freezing occurred, the group would appear inferior. It should be noted that in the present study, all Ss, both experimental and control, performed a very efficient and short latency escape response when shock was not avoided. Thus, the interpretation by Sheffield (1948), in terms of shock-initiated freezing responses, is not applicable.

In addition, Fig. 2 provides the frequency distribution of the anticipatory response latencies for each group in tenths of the warning signal duration, separately for the two acquisition and the extinction days. Not only is the overall frequency of anticipatory response obviously in favor of the instrumental group, even with a well-established escape "unconditioned response," but the modal frequencies are quite different. The escape-only groups responded most frequently just prior to shock onset, whereas the avoidance group during acquisition responded early in the warning interval. There is a consistent shift in modal response latency to that of the respective controls when the avoidance Ss were placed on extinction. Some anticipatory responding must first occur before instrumental reinforcement can be provided, and there would thus appear to be some strengthening of anticipatory responding in the escapeonly condition. The present authors presume this anticipatory tendency to be similar to that manifested in reaction time experiments with a constant fore period-an instrumental response strengthened by effectively minimizing the reaction time (i.e., shock duration) by attempting to predict onset of the shock. In the case of the anticipatory response, the prediction is somewhat inaccurate.

One other point should be made. Although specific data are not available except indirectly in the latency performance of Fig. 2, observations of Ss in testing indicated an obvious casualness about making lever responses. There was no indication of Ss hanging on the lever and making a startled response to the warning signal. Nor were Ss' responses easily interpreted as part of any "innate defensive repertory," as suggested by Bolles $(1967)$. It is believed that an observer would have been hardpressed to detect any essential difference in the manner of responding from that typically found in food reward studies.

In conclusion, the reinterpreted extinction procedure provided extinction data for avoidance responses more in keeping with positive reinforcement studies, with fairly rapid and reliable decreases in responding. Procedures such as discrimination, shaping, and partial reinforcement which have extinction as an essential component can now be explored in avoidance conditioning, problems not capable of realistic evaluation under the traditional definition of avoidance extinction. Such studies are currently being undertaken in our laboratory. The application of the present point of view to escape ex tinction should be obvious, but also has little previous use in the research literature. REFERENCES

BOLLES, R. C. Theory of motivation. New York: Harper \& Row, 1967. BROGDEN, W. J., LIPMAN, E. A., \& CULLER, E. The role of incentive in conditioning and extinction. American Journal of Psychology, $1938,51,109-117$.

D'AMATO, M, R. Role of anticipatory responses in avoidance conditioning; an important control. Psychonomic Science, 1967, 8, 191-192.

KATZEV, R. Extinguishing avoidance responses as a function of delayed warning signal termination. Journal of Experimental Psychology, $1967,75,339-344$.

RESCORLA, R. A., \& SOLOMON, R. L. Two process learning theory: Relationship between Pavlovian conditioning and instrumental conditioning. Psychological Review, 1967, 74, 151-182.

SHEFFIELD, F. D. Avoidance training and the contiguity principle. Journal of Comparative \& Physiological Psychology, 1948, 41, 161-177.

NOTE

1. A preliminary draft of this paper was read at M.P.A. 1968. 